\title{
Unilateral pulmonary vein atresia without anomalous connection in adult patient with recurrent severe hemoptysis
}

\author{
Andrea Dell'Amore, Alessio Campisi, Stefano Congiu, Domenica Giunta, Giampiero Dolci, Fabio Niro \\ Department of Cardiothoracic Surgery, S.Orsola University Hospital, Bologna, Italy \\ Correspondence to: Andrea Dell'Amore. Department of Cardiothoracic Surgery, S.Orsola University Hospital, Via Massarenti 9, Bologna, Italy. \\ Email: andrea.dellamore@aosp.bo.it.
}

\begin{abstract}
Isolated unilateral pulmonary vein atresia (UPVA) is a rare congenital malformation and the management remains controversial. In adults, pneumonectomy is the treatment of choice when significant hemoptysis becomes life-threatening. We report a case of a 28 -year-old male with isolated unilateral right atresia of the pulmonary vein who had life-threatening hemoptysis treated with bronchial arteries embolization followed by successful right pneumonectomy.
\end{abstract}

Keywords: Pulmonary vein atresia; pneumonectomy; hemoptysis; pulmonary vascular disease; congenital

Received: 06 April 2018; Accepted: 27 April 2018; Published: 22 May 2018.

doi: 10.21037 /jovs.2018.05.03

View this article at: http://dx.doi.org/10.21037/jovs.2018.05.03

\section{Introduction}

Unilateral pulmonary vein atresia (UPVA) is the failure of connection between the common pulmonary vein and the left atrium (1). UPVA is a rare congenital malformation that in $32-50 \%$ of cases it is associated with other cardiac malformations $(1,2)$. Isolated UPVA is uncommon, the diagnosis occurs during early childhood because of asthma history, recurrent pulmonary infections, or hemoptysis (1). Diagnosis in adults is unusual, frequently related to hemoptysis and pulmonary hypertension (3-5). In these patients, UPVA is associated with significant morbidity and mortality $(1,2)$.

\section{Case presentation}

A 28-year-old male was referred to our Unit because of discovery of a right pulmonary vein atresia. The patient had recurrent bronchitis since childhood and hemoptysis since the age of 20, which wasn't investigated. In November 2016, the patient was admitted to another hospital because of severe hemoptysis, and treated with bronchial artery embolization which resolved the bronchial bleeding. The diagnosis of UPVA was done after angio-computedtomography (Angio-CT)-scan and echocardiography. Since extensive embolization of the bronchial arteries was

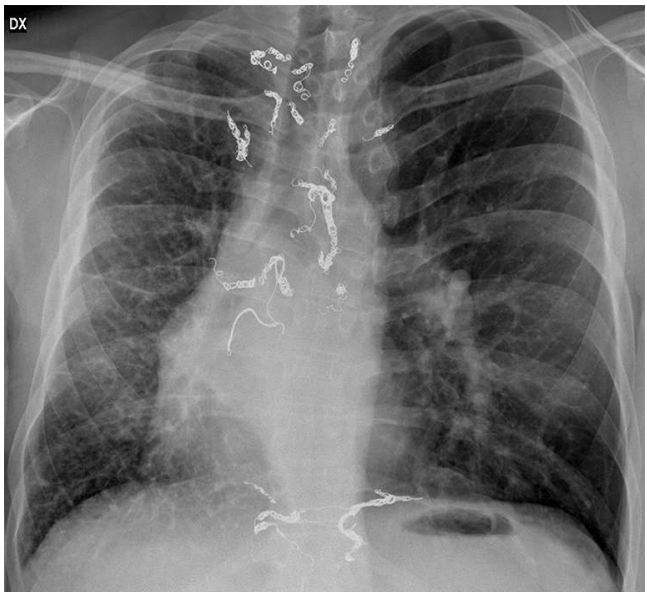

Figure 1 Chest $\mathrm{X}$-ray showing the result of extensive embolization of the bronchial collateral arteries.

done (Figure 1) with resolution of bleeding, they decided a conservative treatment and follow-up. In September 2017, the patient was admitted to the ICU of another hospital because of massive hemoptysis that required selective intubation to avoid suffocation and hemorrhagic shock that was treated with multiple red blood cell transfusions. The bleeding resolved after performing a second emergent selective bronchial embolization. Afterwards, the patient 

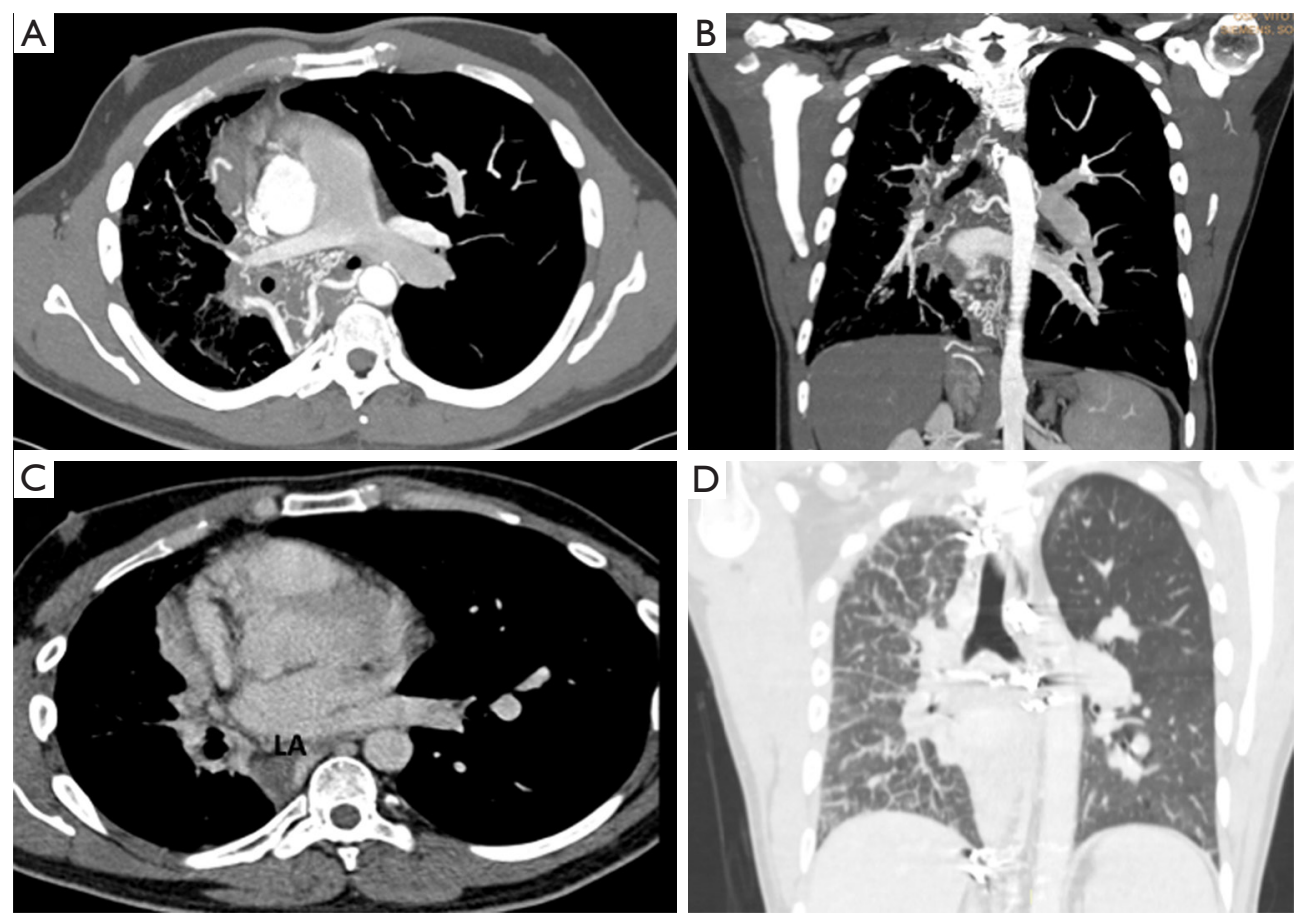

Figure 2 Angio-CT-scan showed a hypoplastic right pulmonary artery with hypertrophic and hyperplastic collateral bronchial circulation $(A, B)$, absence of the right main pulmonary vein (C), diffuse thickening of the right interlobar septa, with small right hemithorax (D). LA, left atrium.

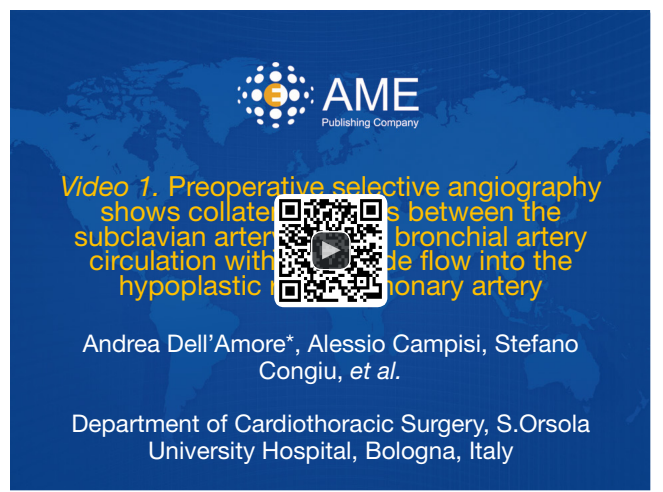

Figure 3 Preoperative selective angiography shows collateral arteries between the subclavian artery and the bronchial artery circulation with retrograde flow into the hypoplastic right pulmonary artery (6). The right pneumonectomy was technically demanding due to the presence of hypervascularized tissue all around the pulmonary hilum. Ultrasonic scalpel and mechanical staplers were used to facilitate the dissection of the hilum and to resect the main pulmonary artery and the right bronchus.

Available online: http://www.asvide.com/article/view/24893 was transferred to our hospital for surgical evaluation.

A new Angio-CT-scan confirmed the absence of right pulmonary veins, a hypoplastic right lung, a small right pulmonary artery and diffuse thickening of the right interlobar septa (Figure 2). Bronchoscopy revealed dilated blood vessels with a hyperemic right bronchial mucosa. Trans-thoracic echocardiography revealed no congenital heart disease and no signs of pulmonary hypertension. Despite the previous embolization, a significant collateral blood flow from large bronchial and intercostal vessels draining into the right pulmonary artery was present. Surgical indication was given because of recurrent lifethreatening hemoptysis despite the conservative strategy. Before the operation we decided to complete the embolization of collateral arteries between the thyrocervical trunk and the subclavian artery to the bronchial artery circulation to minimize the bleeding risk during surgery. The operation was scheduled the day after the embolization, and a right pneumonectomy was done (Figure 3).

The patient was transferred to the intensive-care-unit 
and extubated after 4-hours. The post-operative period was uneventful and he was discharged from the hospital on the 8th postoperative day in optimal medical condition, and the patient is currently asymptomatic.

\section{Discussion}

The management of UPVA remains controversial $(1,4)$. Early diagnosis during infancy can allow an anatomical surgical correction. It would be very important to identify these patients before pulmonary hypertension or massive hemoptysis ensues (1). Unfortunately, when the diagnosis occurs in adulthood, acquired problems make the anatomical correction impossible $(1,2,4)$. In these cases, pneumonectomy is the treatment of choice when recurrent infections or significant hemoptysis become frequent and life-threatening. Moreover, pneumonectomy eliminates the left-to-right shunt and the dead-space with improvement in pulmonary function (5). Despite a clinical history of recurrent pneumonia and hemoptysis, our patient's UPVA was diagnosed only in adulthood following one episode of severe hemoptysis. During the last and life-threatening episode of hemoptysis, the emergent and extensive embolization of the hyperplastic and hypertrophic bronchial circulation allowed to control the bronchial bleeding. Due to the severity of the bronchial bleeding and the extensive collateral bronchial circulation we decided to schedule the patient for a right pneumonectomy. In this case, it's very important to exclude other cardiovascular malformations and severe pulmonary hypertension that could become contraindications for surgery. In UPVA, surgery is particularly demanding because of the high-risk of intraoperative bleeding. Therefore, to minimize the risk of intraoperative hemorrhage, we embolized all the possible collateral arteries to the right lung. The extensive arterial embolization allowed us to perform a dissection of the hilar pulmonary structures with limited blood loss.

In conclusion, treatment of UPVA depends on the age of the patient and clinical manifestations. Pneumonectomy is the treatment of choice in symptomatic adults. Preoperative planning is mandatory to reduce morbidity and mortality. In particular, bronchial arteries embolization may reduce intraoperative bleeding. Surgery is technically demanding but it's the only definitive treatment of isolated UPVA.

\section{Acknowledgements}

None.

\section{Footnote}

Conflicts of Interest: The authors have no conflicts of interest to declare.

Informed Consent: Written informed consent was obtained from the patient for publication of this manuscript and any accompanying images.

\section{References}

1. Pourmoghadam KK, Moore JW, Khan M, et al. Congenital unilateral pulmonary venous atresia: definitive diagnosis and treatment. Pediatr Cardiol 2003;24:73-9.

2. DeMastes-Crabtree CS, Kim E, Morton RL. Unilateral pulmonary vein atresia: A rare case of hemoptysis. Respir Med Case Rep 2015;15:83-4.

3. Heyneman LE, Nolan RL, Harrison JK, et al. Congenital unilateral pulmonary vein atresia: radiologic findings in three adult patients. AJR Am J Roentgenol 2001;177:681-5.

4. Zhang $\mathrm{M}, \mathrm{Wu} \mathrm{QC}$, Zhang $\mathrm{C}$, et al. Isolated unilateral pulmonary vein atresia in adult patients: a case report and literature review. Heart Surg Forum 2010;13:E370-2.

5. Gómez Hernández MT, Rodríguez Pérez M, F Jiménez M. Isolated unilateral pulmonary vein atesia in adults. Arch Bronconeumol 2015;51:424-5.

6. Dell'Amore A, Campisi A, Congiu S, et al. Preoperative selective angiography shows collateral arteries between the subclavian artery and the bronchial artery circulation with retrograde flow into the hypoplastic right pulmonary artery. Asvide 2018;5:519. Available online: http://www. asvide.com/article/view/24893

doi: $10.21037 /$ jovs.2018.05.03

Cite this article as: Dell'Amore A, Campisi A, Congiu S, Giunta D, Dolci G, Niro F. Unilateral pulmonary vein atresia without anomalous connection in adult patient with recurrent severe hemoptysis. J Vis Surg 2018;4:111. 